\title{
Plant Species Classification through New Feature Extraction Model-Velocity Clamping Based Intersecting Cortical Model
}

\author{
I. KirubaRaji, K. K. Thyagharajan
}

\begin{abstract}
Plant classification is an active research area. The purpose of our current work is to develop a suitable feature extraction model. This paper suggests a technique to extract the geometric invariants of leaf images using a new velocity clamping based particle swarm optimized intersecting Cortical Model (VCPSO-ICM). Earlier geometric moments were assessed by transforms, separate normalization was used and they were costly. Intersecting cortical model (ICM) is used to avoid the usage of separate normalization for moment invariants of leaf images. In this model, the image is directly processed, as there is no need for preprocessing images. Parameters used in the intersecting cortical model (ICM) are difficult to set for each image separately. This is solved by our model. Time sequences are extracted from each image based on new parameters. Finally, a neural network is preowned to segregate the species of leaf images. This new feature evaluation model is tested on leaf snap database and results are compared with traditional Pulse Coupled neural network (PCNN), simplified Intersecting Cortical Model (ICM).This model achieves a higher accuracy than the existing methods.
\end{abstract}

Keywords: Intersecting Cortical Model (ICM), Neural Network, Particle swarm optimization (PSO), Pulse Coupled Neural Network (PCNN), Velocity Clamping.

\section{INTRODUCTION}

An intelligent computing system plant classification is an active research area. Due to ecological conditions all plants need to be digitized. A digitized analysis of plants is done by extracting the domain specific features. There are two types of domain specific features narrow and wide. Narrow domain features offer a less changeable features but wide area domain provides high changeable features. Feature extraction plays a vital role in plant classification. Appearance based features such as shape of plants, texture, veins, leaf margin, leaf apex, base of leaf are extracted from plants. Images are generally analyzed by shapes, textures and colors of the leaf. All leaves are in green color and due to some situations; there is damage in shape, textures and color. Geometric moments are acting as important features to access the pattern of leaves. Image transform techniques are

Revised Manuscript Received on November 22, 2019.

* Correspondence Author

I.Kiruba Raji, Department of Computer Science Engineering, R.M.D Engineering College, Kavaraipettai, Chennai, India. Email:kiruba161107@gmail.com

K.K.Thyagharajan, Department of Electronics and Communication Engineering, R.M.D Engineering College, Kavaraipettai, Chennai, India, Email: kkthyagharajan@yahoo.com used to identify moment specific features such as Pyramid Histogram of oriented Gradients (HOG) (Zhao ZQ et al.2015), Polar Fourier Transform (PFT) (Abdul Kadir LE et al.2011), Zernike Moment (Pallavi P et al. 2014), Edge Angle descriptor (EAGLE) (Charters J et al.2014)

\section{RELATED WORK}

The Parameters of Pulse Coupled Neural Network is considerable part in pattern classification, as parameters of same value are not suitable for all images. They need to be adjusted according to biological characteristics of neural network. Manual adjustment of parameters by trial and error method utilizes more time; the parameters need to be optimized. There are three different types of parameter augmentation techniques (Xinzheng $\mathrm{Xu}$ et al, 2016) 1.Determine PCNN parameters based on firing mechanism of images. 2. Combine PCNN properties and image characters.3.Intelligent Optimization Methods.

Parameters are determined based on firing mechanism of pulse coupled neural networks. Theoretical analysis is needed for representing dynamic behavior of PCNN, which affects the network parameters of simplified PCNN model (Deng Xiang-yu et al. 2012, 2014). It is applicable only for image detachment and edge detection. Image characteristics such as gray level histogram, gradient energy, mean intensity value (Kandasamy Kondampatti Thyagharajan et al, 2018) are widely used in PCNN parameter determination, it is unnatural. Manual adjustment of some parameters is needed and it is applicable for image segmentation (Thejaswi H.Raya et al, 2011, Heba.F.Eid et al, 2018) and image fusion. Intelligent Optimization methods automatically adjust the parameters there is no needed to use human interaction to adjust the constant of PCNN model. It is applicable for feature extraction.

Ilige S.Hage et al (2013) adopted pulse coupled neural network with particle swarm optimization to enhance the parameters of PCNN to automatically segment cortical bones by using maximum entropy and energy until it reaches a maximum threshold. Mona Mahrous Mohammed (2015) et al. used PCNN and GA for image retrieval and classification of images. It takes more computational time, as PCNN constants are optimized by setting a pretend genesis as 300 and it generates one dimensional size of feature vector as length of 70.Xu Xinzheng (2011) used PCNN PSO for image segmentation and achieved good segmentation results on noisy images. Linlin Mu et al (2013) adopted quantum based particle swarm optimization for determining automatic parameters 
for pulse coupled neural network to segment the images.

Zhao bin Wang et al (2015) have used Pulse Coupled neural network entropy sequence with morphological features and has achieved more than $90 \%$ accuracy on leaf data bases. Zhao bin Wang et al (2017) have used Dual Pulse Coupled network entropy sequence to generate for each local region of pulse images and classified it using Bag of visual Words model. It performs well on noisy images and has achieved the highest recognition accuracy as $97.63 \%$, but it takes more computational time because of its complexity. They use the same values for constants of PCNN. It is not suitable technique, if there is a change in the images.

There are many techniques used for leaf feature extraction, they are shape, texture and margin leaf base as features. The accuracies are not satisfactory. A new method of optimized feature extraction technique is developed based on velocity clamping based Particle swarm optimization (PSO)-Intersecting Cortical Model. It works well on bright pixels, it is robust to noise, illumination, translation, rotation and scale invariance. It is applicable for single leaf images. It works on images without sharp edges and straight lines, when compared to PCNN model it has less number of equations, two stage oscillator models and computationally fast. Parameters of ICM are differed from image to image. Due to the extraction of the correct features of image parameters used in ICM are optimized. PSO is used as a parameter optimization technique because of its performance and less time computation.

The paper is structured as follows section 2 describes the basic methods and materials. Section 3 describes the feature extraction methods. Section 4 provides the experimental results and discussions based on the evaluation of leaf snap databases. Section 5 deals with the conclusion of the paper.

\section{MATERIALS AND METHODS}

The pulse-coupled neural network has two inputs, a linking neuron and a feeding neuron, that generates pulse images based on time. The result produced by this method is better than that of others even though it takes a long computation time due to its sequential nature. The first state of oscillator is a neuron state and the second state is the dynamic threshold. Ulf Ekblad and Jason M. Kinser(2005) is derived a intersecting cortical model with the condensed mathematical statements from the PCNN to provide good inter-neuron communication. It is designed from several visual cortex models. It is widely used for image processing fields such as change detection, motion estimation, and image enhancement, feature extraction.

The ICM equations are,

$$
\begin{aligned}
& F_{i j}(n)=f F_{i j}(n-1)+\sum_{i j} W_{i j} Y_{i j}(n-1)+S_{i j} \\
& \theta_{i j}(n)=g \theta_{i j}(n-1)+h Y_{i j}(n-1) \\
& Y_{i j}(n)= \begin{cases}1 & \text { if } F_{i j}(n)>\theta i j(n) \\
0 & \text { otherwise }\end{cases}
\end{aligned}
$$

In the above equation, $S_{i j}$ is the input stimulus (input image, scaled to 1.0), $\mathrm{Y}_{\mathrm{ij}}$ is the firing state of the neuron (output image), and $\mathrm{f}, \mathrm{g}$, and $\mathrm{h}$ are scalar values. The values of $f, g$ and $h$ are $0.9,0.8$, and 20. $\mathrm{n}$ is the number of iterations in between $1 \ldots \mathrm{N}$ and $\mathrm{f}, \mathrm{g}$ are decay constants. The Fij turns after the longest period of $\theta \mathrm{ij}$, so $\mathrm{f}>\mathrm{g}$. $\mathrm{W}_{\mathrm{ij}}$ is the interconnection of neurons. It follows inverse square rule ( Ulf Ekblad et al.(2004)). The smoothing function is used to determine the connection between the neighboring pixels of the neurons. $\mathrm{Y}_{\mathrm{ij}}$ is the binary pulse image of the output of the neurons. The neuronal structure of the intersecting cortical model is shown in Fig1.

\section{A. Optimized Intersecting Cortical Model}

There are three parameters in the intersecting cortical model: the two decay constants $\mathrm{f}$ and $\mathrm{g}$, the scalar value $\mathrm{h}$. and it is a challenge to adjust the values of these parameters for all types of input images. After the input images are changed, the values of the parameters are unsuitable for new images. Different initial values of decay constants and scalar values are used to exhaust the features of images to efficiently utilize the biological characteristics of the ICM. Xinzheng $\mathrm{Zu}$ et al. (2016) is identified three ways to optimize the parameters, to determine whether the parameters are adaptive, combining the neuron model's properties with image characteristics, and use swarm based optimization methods to regulate the absolute values of the parameters, depending on the input image.

The swarm based optimization method is efficient because it excludes the inconvenience of trial-and-error parameter settings. The optimized ICM is used to improve the signature quality of the images. Consequently, the ICM is functionally modified with an automatic parameter setting model with particle swarm optimization.

\section{B. Particle Swarm Optimization}

The Particle Swarm Optimization (PSO) is a population-based technique built on the social behavior of fishes in schools and birds in flocks. There are two types of PSO. Continuous PSO works well on discrete domain search space. Parameter Optimization of Intersecting Cortical model operates on continuous search space, as it updates decay constants and scalar value between lower and upper bound values. Binary PSO works well on search space $(0,1)$ and update the particles position. It is useful in feature selection, pattern matching. PSO is applied continuously for parameter Optimization. James Kennedy (1994) initiated Particle Swarm Optimization (PSO) by utilizing the concepts governing the social and cognitive behavior of fishes and birds. It is a particularly worn problem-solving approach in engineering, where the universal and actual sociometric behavior of an individual is examined in terms of evaluation, comparison and imitation. Two parameters are used in the PSO: $g_{\text {best }}$ and $\mathrm{l}_{\text {best }}$ (James Kennedy, 2001 and Davoud Sedighizadẹh)et al., 2009).

The $\mathrm{g}_{\text {best }}$ stands for the global best. It interconnects an entije population; one to another. The $1_{\text {best }}$ indicates the local best. It creates a neighborhood for each member of the population and itself. Compared to the Genetic Algorithm (Mona Mahrous Mohammed et al., 2015) parameter values are quickly adjusted by PSO.( Xinzheng Xu et al., 2011, IIige S. Hage et al.,2013, Linlin Mu et al, 2013, Weng Chun Tan et 
al., 2016, Kehan Gao et al,2012).

\section{Parameter selection}

The parameters used in the PSO are the fitness function, dimension of the particle, population size, inertia factor, and terminal condition maximum iteration.

1. Population size: It performs a key role in the evolutionary algorithm. Total numbers of candidate solutions are called as population or swarm. A small size of the population may result in less aggregation while a large size of population one increases the computational effort. A population size of [5-25] is chosen (Yanijiang Miao et al. (2009)). Population size: 5, needs to optimize only 3 parameters.

2. The fitness function is based on entropy as it gives information on the quality of image and counts the number of ones and zeros. The entropy of a discrete random variable $\mathrm{X}$ that has a probability distribution $\mathrm{P}_{\mathrm{x}}=\left(\mathrm{P}_{1}, \mathrm{P}_{2}, \ldots \mathrm{P}_{\mathrm{n}}\right)$ is defined as

Therefore, image entropy is used as a fitness function ( Yide Ma et al., 2010).

$E(I)=-P(0) \ln P(0)-P(1) \ln P(1)$

fitvalue $=\max (E(I))$

The probability of pixel value ' 1 ' i.e. $\mathrm{P}(1)$ of a binary image is defined as the ratio of number of $1 \mathrm{~s}$ in the image. Similarly $\mathrm{P}(0)$ is the probability of pixel value ' 0 ' and it is defined as the ratio of number of $0 \mathrm{~s}$ in the binary image.

3. In a PSO, each candidate solution is called as particle in $\mathrm{D}$ dimensional search space. D refers to the number of parameters to be optimized.

The parameters are decay constants $\mathrm{f}$ and $\mathrm{g}$ and scalar values (h). From our experiment $\mathrm{D}=3$

4. The inertia factor (Laura Lanzarini et al. 2011) indicates the elasticity of particle that moves in the search space. It is used to adjust the movements of particles in the search space. Large values make large movements and small values make fine adjustments $(w=1)$

5. Terminal condition: Maximum number of iterations

\section{Velocity Clamping Based PSO-Algorithm}

Input: Swarm size $(S)=5$, Dimension of the Particle $(D)=3$, Inertia factor $=0.5$,

Social Component $(\mathrm{c} 1)=1$, Cognitive Component $(\mathrm{c} 2)=1$, $\mathrm{lb}=[0.2,0.1,1], \mathrm{ub}=[0.9,0.8,20]$,

\section{Algorithm:}

$\mathrm{x}[\mathrm{f}, \mathrm{g}, \mathrm{h}]=$ Position of particle, $\mathrm{v}[\mathrm{f}, \mathrm{g}, \mathrm{h}]=$ velocity of Particle $\mathrm{fp}=$ Best Particle Function values $(\mathrm{S})$

$\mathrm{p}_{\mathrm{id}}=$ Individual Best Particle Position, $\mathrm{p}_{\mathrm{gd}}=$ Global Best Particle Position,fg=Starting Value of swarm

For each swarm $\mathrm{i}=1: \mathrm{S}$

For each dimension $\mathrm{j}=1: \mathrm{D}$ :

Initialize Particle's Position

$x_{i, 0}(f, g, h)=v b(f, g, h)+x_{i, y}(f, g, h) *(u b(f, g, h)-l b(f, g$ Initialize Velocity

$$
\begin{aligned}
& v_{\mathrm{i}, 0}(f, g, h)=\operatorname{vlow}(f, g, h)+\text { mean * } \\
& (\operatorname{vhigh}(f, g, h)-\operatorname{vlow}(f, g, h))
\end{aligned}
$$

Calculate fitness value based on VCPSO Eq(4,5)

End For //Dimension D

End For //Swarm

//Iteration

For iteration $\mathrm{i}=1: 10$ :

For each particle $\mathrm{i}$ in $1: \mathrm{S}$

For each Dimension $\mathrm{j}$ in 1:D

Update velocity and Position of the particle

$$
\begin{aligned}
& v_{\text {id } i+1}(f, g, h)=w * v_{i d, i-1}(f, g, h)+c 1 r 1 * \\
& \left(p_{i d_{i}-1}(f, g, h)-x_{i d_{i}-1}(f, g, h)\right)+c 2 r 2 * \\
& \left(p_{g d_{i}-1}(f, g, h)-\right. \\
& \left.x_{\text {id } i-1}(f, g, h)\right)
\end{aligned}
$$

$$
x_{i d, i+1}(f, g, h)=x_{i d i-1}(f, g, h)
$$

$+v_{i d i-1}(f, g, h)$

(4) Where $v_{\mathrm{i} d_{i+1}}$ is the $\mathrm{i}^{\text {th }}$ particle velocity in iteration $\mathrm{i}+1, w$ the inertia factor, $c 1, c 2$ the constant weight vectors, r1,r2 the random factors $[0,1]$, $p_{i d_{j}, i-1}, p_{g d_{i} i-1}$ the best position of individual particle and the neighborhood particle, and $x_{i d i+1}$ the particle position in iteration $i+1$.

\# Calculate Fitness value

If $f x<p_{i d, i}(f, g, h)$ :

Set Current entropy as a fitness value

$$
\text { End if }
$$

If $f x<p_{g d, i}(f, g, h)$ :

Select the particle as a global best particle End if

End For //Dimension D

End for // Each Swarm S

End For//Maximum Iteration

Output: Best Swarm Position (g) , Fitness function Value(minimum distance value to reach the

$$
\theta_{i j}(n) \text {-internal activation unit }
$$

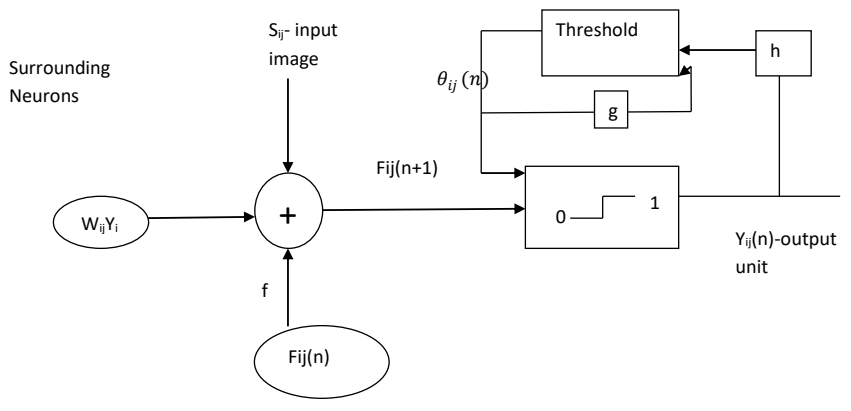

Fig. 1. Neuron Structure of ICM

\section{IV.EXPERIMENTAL RESULTS AND DISCUSSIONS}

The experiment was conducted on leaf snap database. The leaf snap database was downloaded from http://leafsnap.com Lab images, 
consists of high-quality images of the pressed leaves from the Smithsonian collection. Several samples of the species with images appear in controlled back-lit and front-lit versions. The field images consisting of 7719 "typical" images taken on mobile devices (mostly iPhones) in outdoor environments, and contain varying degrees of blur, noise, illumination patterns, and shadows. All 185 tree species from the Northeastern United States are covered in the dataset. There are 10 species from the Leaf snap database have been selected which are similar and different in shape, both in inter- and intra classes.

The different species of leaf snap database is classified through neural network classifier. The different classifiers used include the K-nearest neighbor, Bayesian, the ensemble-based. The performance of the classifiers is affected by the type, size and quality of the data. Several researchers have suggested the use of a neural network classifier for classifying leaf species . A pattern net is worn to distinguish the patterns.

The performance matrix is calculated from the confusion matrix is as the follows:

Accuracy=True Positive+True Negative/Total Images

Table 1: Confusion Matrix

\begin{tabular}{|c|c|c|c|c|c|c|c|c|c|c|}
\hline $\begin{array}{l}\text { class } \\
\text { Label }\end{array}$ & 1 & 2 & 3 & 4 & 5 & 6 & 7 & 8 & 9 & 10 \\
\hline 1 & 33 & 1 & 0 & 0 & 0 & 0 & 0 & 2 & 0 & 0 \\
\hline 2 & 1 & 29 & 0 & 0 & 0 & 0 & 0 & 1 & 0 & 0 \\
\hline 3 & 0 & 0 & 31 & 2 & 0 & 0 & 0 & 1 & 0 & 0 \\
\hline 4 & 1 & 0 & 3 & 37 & 0 & 1 & 0 & 1 & 0 & 0 \\
\hline 5 & 0 & 0 & 0 & 0 & 17 & 2 & 1 & 0 & 0 & 0 \\
\hline 6 & 0 & 0 & 0 & 0 & 0 & 70 & 0 & 2 & 0 & 0 \\
\hline 7 & 0 & 0 & 0 & 0 & 1 & 2 & 25 & 0 & 0 & 0 \\
\hline 8 & 1 & 1 & 2 & 4 & 1 & 0 & 0 & 32 & 0 & 1 \\
\hline 9 & 0 & 0 & 0 & 0 & 0 & 0 & 0 & 1 & 14 & 0 \\
\hline 10 & 0 & 0 & 0 & 0 & 0 & 0 & 0 & 0 & 0 & 51 \\
\hline \multicolumn{5}{|c|}{ Overall accuracy } & 91 & & & & & \\
\hline
\end{tabular}

The receiver operating Characteristics curve(ROC) has describe the relationship between True Positive rate and False Positive Rate. In ROC Curve the diagonal line $(0,1)$ indicates perfect classification. The classes classified above the diagonal has provide better classification. The figures 2,3 and 4 shown all the classes provide better classification results in training set compared to test set.

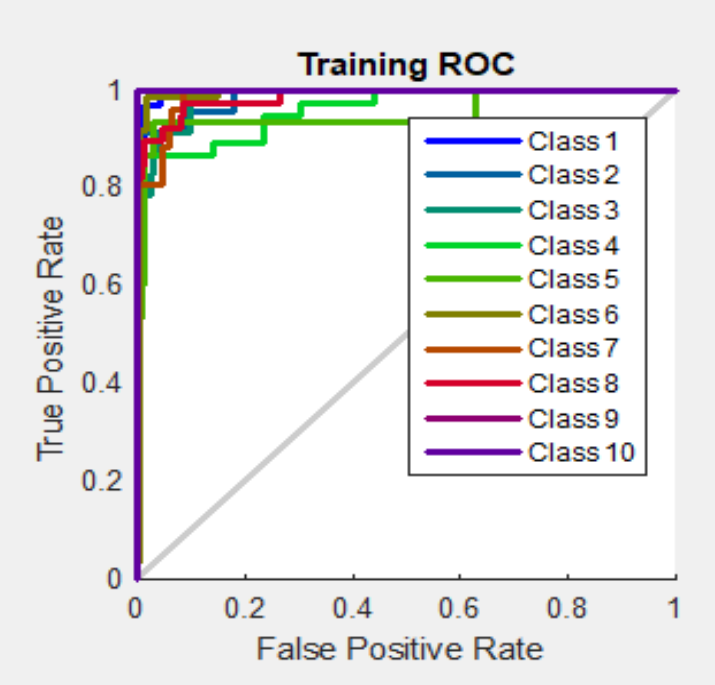

Fig 2. ROC Curve of Training set

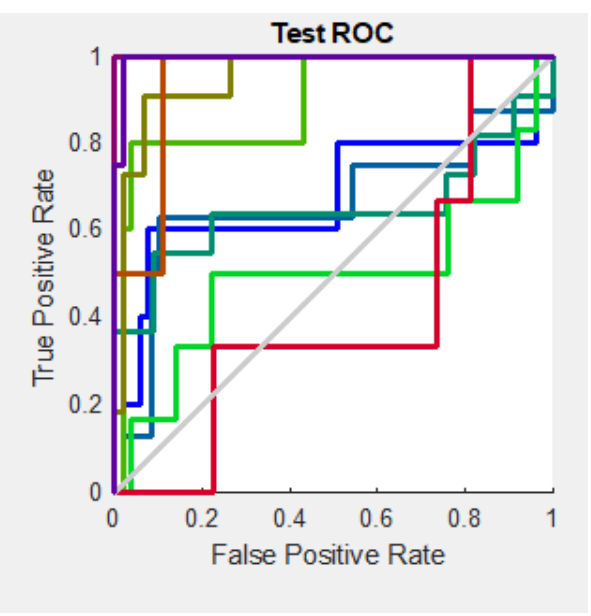

Fig 3.ROC Curve of Test Set

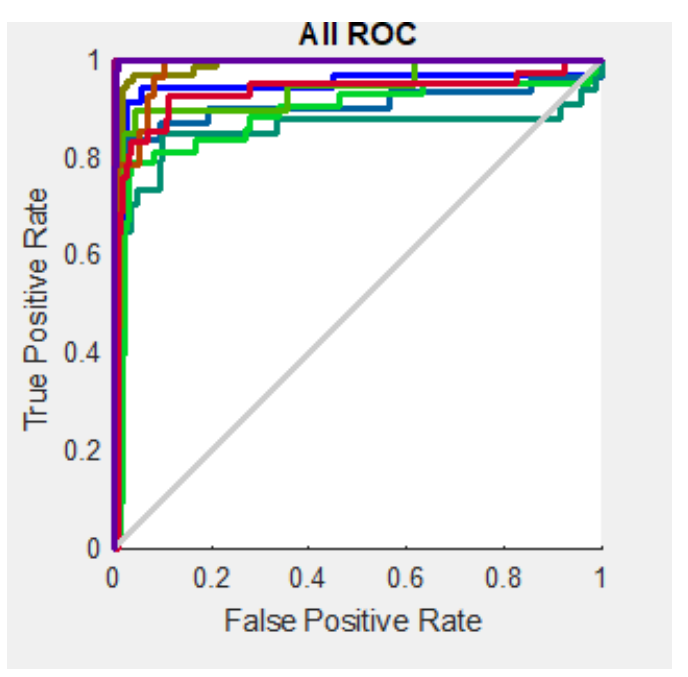

Fig 4. ROC Curve of all images

\section{CONCLUSION}

A new feature extraction method has proposed in this paper based on ICM and PSO. ICM is deployed to extract the correct features of the leaf images. This method is used to detect distinct objects because of their pulse simulations. There is no need for prior training of the images. Previously used methods were needed to separate dimension reduction techniques. However, it can control the dimensions of the feature vectors. In our model, We concluded that the size of the feature vector is 34 where the first 30 values had obtained from time sequence, 3 values from the particle's position $\mathrm{f}, \mathrm{g}, \mathrm{h}$, and fitness value of particle. The size of the feature vector when it is less, we have acquired the highest classification accuracy of $91.12 \%$. This model is used in change detection to identify the changes in a particular region. In the future, we can train our model to identify the leaf diseases, which is computationally fast. 


\section{REFERNCES}

1. Abdul Kadir LE, Susanto NA, Santosa PI (2011) A comparative experiment of several shape methods in recognizing plants. Int J Comput Sci Inf Technol 3(5):256-263. https://doi.org/10.5121/ ijcsit.2011.3318

2. AP Engelbrecht(2014). Fitness Function Evaluations:A Fair Stopping Condition?. IEEE Symposium on Swarm Intelligence.doi: 10.1109/SIS.2014.7011793

3. Charters J, Wang Z, Chi Z, Tsoi AC, Feng DD (2014) Eagle: a novel descriptor for identifying plant species using leaf lamina vascular features. In: 2014 IEEE international conference on multimedia and expo workshops (ICMEW), pp 1-6. https://doi org/10.1109/icmew.2014.6890557

4. Davoud Sedighizadeh, EllipsMasehian (2009). Particle Swarm Optimization Methods, Taxonomy and Applications. International Journal of Computer Theory and Engineering, 1(5),1793-8201.doi: 10.7763/IJCTE.2009.V1.80

5. DENG XiAnG-YU, Yide Ma. (2012). PCNN Model AutOMATiC PARAMETERS DETERMINATION AND ITS MODIFIED MODEL. ACTA ELECTRONICA SINICA. $\quad 5.955-964$ 10.3969/J.ISSN.0372-2112.2012.05.015.

6. DENG XIANG-YU, YIDE MA(2014). PCNN MODEL ANALYSIS AND ITS AuTOMATIC PARAMETERS DETERMINATION IN IMAGE SEGMENTATION AND EDGE DETECTION. CHINESE JOURNAL OF ELECTRONICS 23(1):97-103.

7. Heba F.Eid, Ajith Abraham (2018).Plant Species identification using leaf biometrics and swarm optimisation: A hybrid PSO, GWO, SVM Model.International Journal of hybrid Intelligent System.(1).pp:1-11.

8. Ilige S. Hage, Ramsey F.Hamade(2013). Segmentation of Histology slides of cortical bone using pulse Coupled neural networks optimized by particle swarm optimisation. Computerized Medical Imaging and Graphics.37.pp:466-474.

9. James Kennedy, Russel Elberhatt(1994), Particle Swarm Optimization, 1995. Proceedings., IEEE International Conference on Neural Networks.1942-1948. doi: 10.1109/ICNN.1995.488968

10. Kandasamy Kondampatti Thyagharajan,Governor Kalaiarasi(2018). Pulse Coupled neural network based near duplicate detection of images(PCNN-NDD). Advances in Electrical and Computer Engineering.18(3):pp.87-96.

11. Kehan GaO, Haibin Duan, Yan Xu, Yang Zhang, ZhuOShu L (2012). ARTIFICIAL BEE COLONY APPROACH TO PARAMETERS OPTIMIZATION OF PUlSE COUPLED NEURAL NETWORKS. IEEE 10TH INTERNATIONAL CONFERENCE ON INDUSTRIAL INFORMATICS .128-132.DOI:10.1109/INDIN.2012.6301362

12. Laura Lanzarini,Victoria Leza,Armando De Giusti(2008). Particle swarm optimization with variable population size, Proceedings of Artificial Intelligence and Soft Computing.438-449.doi: $10.1007 / 978-3-540-69731-2 \quad 43$

13. Leaf snap database:http://leafsnap.com. Last visited:22-05-2018

14. Linlin Mu, Maozheng Zhao, chaozhu Zhang (2013). Quantum Particle Optimisation based on chaotic mutation for automatic parameters determination of pulse coupled neural network.Int.J.Computing Science and Mathematics.4(4).pp.354-362.

15. Mona Mahrous Mohammed, Amr Badr, M.B.Abdelhalim(2015). Image Classification and retrieval using optimized pulse Coupled neural network.Expert System with applications.

16. Pallavi P, Veena D (2014) Leaf recognition based on feature extraction and Zernike moments. Int J Innov Res Comput Commun Eng, 67-73. ISSN:2320-09801

17. Thejaswi H.Raya, Vineetha Bettiah, Heggere S.Ranganath (2011) Adaptive Pulse Coupled Neural Networks Parameter for Image segmentation. International Journal of Computer and Information Engineering, 5(1):pp.90-96.

18. Thomas Linblad, Jason M. Kinser (2005). Image processing using Pulse Coupled Neural Networks, Springer, Berlin.Verlag.doi: 10.1007/3-540-28293-9

19. Ulf Ekblad, Jason M. Kinser (2004). Theoretical foundation of the intersecting cortical model and its use for change detection of aircraft, cars, and nuclear explosion tests, Signal Processing ,84(7). 1131 - 1146 doi: 10.1016/j.sigpro.2004.03.012

20. Ulf Ekblad, Jason M. Kinser, Jenny Atmer, Nils Zetterlund(2004). The intersecting cortical model in image processing, Nuclear Instruments and Methods in Physics Research A 525(1-2) , 392-396.doi: 10.1016/j.nima.2004.03.102
21. Weng Chun Tan, Nor Ashidi Mat Isa(2016). Automated SPERM HEAD DETECTION USING INTERSECTING CORTICAL MODEL OPTIMIZED BY PARTICLE SWARM OPTIMIZATION, PLOS ONE.11(9).1-26.DOI: DOI.ORG/10.1371/JOURNAL.PONE.0162985

22. Xinzheng $\mathrm{Xu}$, Shifei Ding, Zhongzhi Shi (2011). Particle Swarm Optimisation for Automatic Parameter Determination of Pulse Coupled Neural Network. Journal of Computers. 6(8).pp.1546-1553.

23. YANJiANG MiaO, ZhiHUA CUI, JiANChaO ZENG (2009). DyNAMiC POPULATION-BASED PARTICLE SWARM OPTIMIZATION COMBINED WITH CROSSOVER OPERATOR. NINTH INTERNATIONAL CONFERENCE ON HYBRID INTELLIGENT SYSTEMS.DOI:10.1109/HIS.2009.84

24. Yide Ma, Li Liu , Kun Zhan, Yongqing Wu (2010) Pulse-coupled neural networks and one-class support vector machines for geometry invariant texture retrieval. Image and Vision Computing 28(11) 1524-1529.doi: 10.1016/j.imavis.2010.03.006

25. Zhao ZQ, Ma L-H, Chen Y, Wu X, Tang Y, Chen CLP (2015) ApLeaf: an efficient android based leaf identification system. Neurocomputing 151:1112-1119. https://doi.org/10.1016/jj.neu com.2014.02.077

26. Zhaobin Wang, Xiaoguang Sun, Zekun Yang, Yaonan Zhang, Ying Zhu, Yide Ma(2017). Leaf Recognition based on PCNN and BOW.Neural Process Letters.

27. Zhaobin Wang, Xiaoguang Sun, Zekun Yang,Yaonan Zhang, Ying Zhu, Yide Ma(2015). Leaf Recognition based on PCNN.Neural Computers and Applications

28. Zulkifli Z, Saad P, Mohtar IA (2011) Plant leaf identification using moment invariants \& general regression neural network. In: 201111 th International conference on hybrid intelligent systems (HIS), pp 430-435. https://doi.org/10.1109/his.2011. 6122144.

\section{AUTHORS PROFILE}

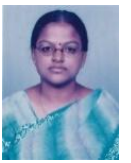

Ms.I. Kiruba Raji, B.E, M.E, (PhD), is an Assistant Professor in Department of Information Technology, since June 2010. She obtained her B.E (CSE) from P.S.N.A College of Engineering \& Technology and M.E (Computer and Communication) from P.S.N.A College of Engineering \& Technology .She is currently pursuing her $\mathrm{PhD}$ (An analysis of automatic Recognition of Herbal Leaves) at Anna University, Chennai. She has been in the teaching profession for the past 10 years. She has published 6 papers in various International Journals and Conferences. She has attended many workshops \& FDPs sponsored by AICTE related to her area of interest. She is a life time members of ISTE.

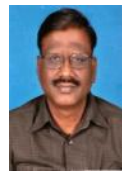

K .K. Thyagharajan B.E,M.E,Ph.D, received his B.Eng degree in Electrical and Electronics Engineering from PSG College of Technology, Madras University, India and received his M.Eng. degree in Applied Electronics from Coimbatore Institute of Technology, India in 1988. He also possesses a Post Graduate Diploma in Computer Applications from Bharathiar University, India. He obtained his Ph.D. degree in Information and Communication Engineering from College of Engineering Guindy, Anna University, India. $\mathrm{He}$ is in teaching profession for around three decades and served at various levels including Principal, Professor and Dean at various Engineering Colleges. He has written 5 books in Computing including "Flash MX 2004" published by McGraw Hill (India), which has been recommended as text and reference book by universities. He is a grant recipient of Tamil Nadu State Council for Science and Technology. He has been invited as chairperson and delivered special lectures in many National and International conferences and workshops. He is reviewer and editorial board member for many International Journals and Conferences. He is a recognized supervisor for $\mathrm{Ph} . \mathrm{D}$ candidates and Master students at Anna University. He has published more than 90 papers in National \& International Journals and Conferences. His research interests include Computer Vision, Semantic Web, Image \& Video Processing, Multimedia Streaming and e-learning. He is a life member of ISTE, CSI INDIA and also senior member and invited member in many professional associations. $\mathrm{He}$ has been recognized by Marquis Who's Who in the World for his contribution to the technical society and his biography has been published in its 25th Anniversary Edition. Personal. 